\title{
An assessment of the current diagnostic criteria for infective endocarditis
}

\author{
Albert W Chan MD FRCPC, Heather J Ross MD FRCPC
}

\begin{abstract}
AW Chan, HJ Ross. An assessment of the current diagnostic criteria for infective endocarditis. Can J Infect Dis 1998;9(4):235-239.

A firm diagnosis of infective endocarditis is difficult to establish. Clinicians often face a decision-making dilemma about whether to treat a patient presenting with some features that suggest endocarditis. This article summarizes both the previous and the recently proposed criteria for the diagnosis of infective endocarditis, and reviews the efficacy of the new criteria. The authors also discuss the experience at The Toronto Hospital, Toronto, Ontario, with this disease, paying specific attention to the application of the new criteria in the diagnostic process.
\end{abstract}

Key Words: Diagnosis, Guidelines, Infective endocarditis

\section{Évaluation des critères actuels en vue du diagnostic de l'endocardite infectieuse}

RÉSUMÉ : Le diagnostic formel de l'endocardite infectieuse est difficile à confirmer. Les cliniciens font souvent face à un dilemme lorsque vient le temps de traiter un patient qui présente certains signes évocateurs de l'endocardite. Cet article résume les anciens critères et les nouveaux critères proposés pour le diagnostic de l'endocardite infectieuse et passe en revue les avantages de ces nouveaux critères. Les auteurs décrivent également l'expérience menée au Toronto Hospital de Toronto, en Ontario, à propos de cette maladie en accordant une attention spécifique à l'application de nouveaux critères dans le processus diagnostique.

I nfective endocarditis (IE) is a condition in which microbial infection occurs on the endothelial surface of the heart. It is characterized by the formation of valvular vegetations, fenestration, abscess and dehiscence of prosthetic valves. Patients with IE may develop fever, malaise, weight loss, anorexia, and symptoms of immunological and embolical phenomenon. However, because of the protean manifestations of IE and the inaccessibility of the involved organ, the disease is often diagnosed with uncertainty based on clinical manifestations. For example, a new onset of a prolonged PR interval on an electro- cardiogram may be indirect evidence of myocardial abscess, but this method is limited by its low sensitivity. The diagnosis of IE is never absolutely certain until explanted valves are assessed pathologically or an autopsy is available. There have been very few published guidelines that assist in the diagnosis of IE. Newly trained physicians often associate the presence of a febrile illness in a patient with a prosthetic heart valve with endocarditis and may subject patients to unnecessary invasive studies and therapy. Should all patients who have prosthetic heart valves and fever undergo an echocardio-

Division of Cardiology, Department of Medicine, The Toronto Hospital, University of Toronto, Toronto, Ontario

Correspondence: Dr Albert W Chan, Vancouver Hospital and Health Science Center, 855 West 12th Avenue, Vancouver, British Columbia V5Z 1M9. Telephone604-232-0030, fax604-232-0080, e-mail achan69@aol.com 


\section{TABLE 1}

\section{von Reyn criteria for diagnosis of infective endocarditis}

\section{Definitive}

Direct evidence of infective endocarditis based on histology from surgery or autopsy, or on bacteriology (Gram stain or culture) of valvular vegetation or peripheral embolus

\section{Probable}

A. Persistently positive blood cultures* plus one of the following: new regurgitant murmur predisposing heart disease $^{\dagger}$ and vascular phenomena ${ }^{\ddagger}$

B. Negative or intermittently positive blood cultures " plus three of the following:

fever

new regurgitant murmur

vascular phenomena

\section{Possible}

A. Persistently positive blood cultures, plus one of the following: predisposing heart disease vascular phenomena

B. Negative or intermittently positive blood cultures with all three of the following:

fever

predisposing heart disease

vascular phenomena

C. For Viridans streptococcus cases only: at least two positive blood cultures without an extracardiac source and fever

\section{Rejected}

A. Endocarditis unlikely, alternative diagnosis generally apparent

B. Endocarditis likely, empiric antibiotic therapy warranted

C. Culture negative endocarditis diagnosed clinically but excluded by postmortem

*At least two blood cultures obtained, with two of two positive, three of three positive, or at least $70 \%$ of cultures positive if four or more cultures obtained; ${ }^{\dagger}$ Definite valvular or congenital heart disease or a cardiac prosthesis (excluding permanent pacemakers); ${ }^{*}$ Petechiae, splinter hemorrhages, conjunctival hemorrhages, Roth spots, Osler's nodes, Janeway lesions, aseptic meningitis, glomerulonephritis, and pulmonary, central nervous system, coronary or peripheral emboli; ${ }^{\S}$ Any rate of blood culture positivity that does not meet the definition of persistently positive. Adapted from reference 2 with permission of the publisher Excerpta Medica Inc

graphic study to evaluate the possibility of IE? Who should be treated with a full course of antibiotics for suspected IE? The purpose of this article is to review the diagnostic criteria used to diagnosis IE.

\section{VON REYN CRITERIA}

In 1981, von Reyn et al (1) published clinical pathological criteria for classifying patients suspected of having IE. The criteria classified cases into definite, probable, possible and rejected endocarditis (Table 1). These criteria have been criticized in recent years for a variety of reasons. First, pathological confirmation is necessary to designate a case as definite IE. Because only some patients require surgery, a substantial portion of cases are classified as 'possible IE', and some of them rejected. Second, the criteria do not incorporate information provided by echocardiography, which plays a critical role in the assessment of patients with suspected endocarditis. Third, they do not consider intravenous drug abus-
TABLE 2

Duke criteria for diagnosis of infective endocarditis

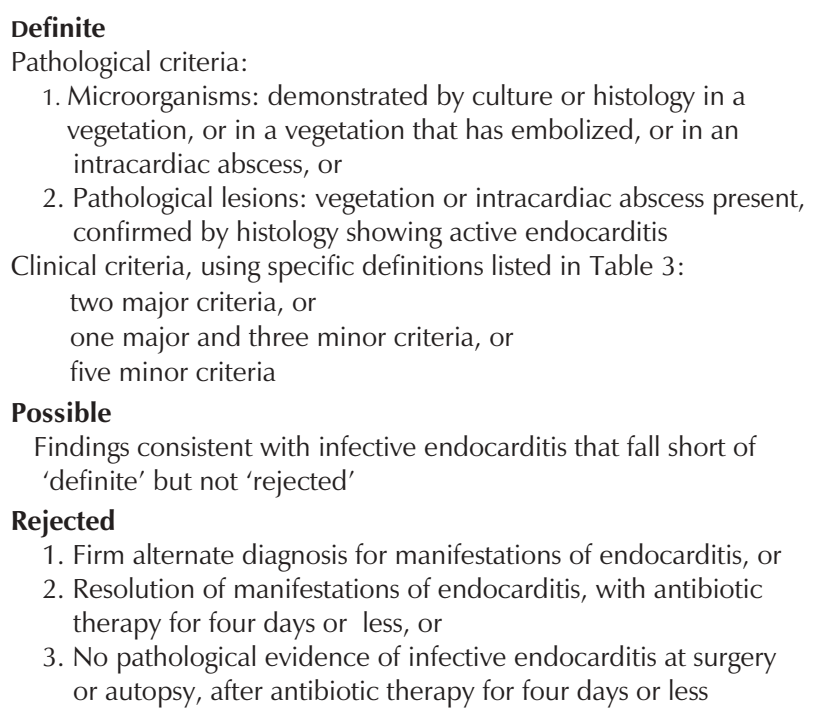

Adapted from reference 2 with permission of Excerpta Medica Inc

ers as high risk and predisposed to IE due to needle-sharing. Because of these criticisms, there is a need for a more comprehensive set of guidelines for diagnosing IE.

\section{DUKE CRITERIA}

Durack et al (2) from Duke University, Durham, North Carolina introduced a new set of criteria that incorporated findings from the two-dimensional echocardiogram and a history of intravenous drug use into the clinical assessment. The new criteria were modelled after the Jones criteria (3) used for the identification of cases of rheumatic fever.

The proposed Duke criteria defined cases of suspected IE into definite, probable and rejected (Table 2). 'Definite IE' was intended to identify patients with a very high likelihood of having IE. Cases for which pathological confirmation is available are classified into this category. On the other hand, because only one-third of patients with native valve IE undergo surgery or autopsy during the acute illness, cases that fulfil certain clinical parameters that are considered typical of IE are also included in this category (Table 3). The 'rejected IE' group comprises cases in which the syndrome resolves spontaneously with a short course of antibiotic therapy or when an alternate diagnosis that explains the presentation is made. Cases that belong to the 'possible IE' category are those that do not meet all the criteria for 'definite IE' and yet manifest findings that are sufficiently suggestive of IE to make rejection of the diagnosis clinically unjustified.

The clinical features of IE are separated into two major and five minor criteria under the Duke criteria. Cases that have two major, one major plus three minor, or five minor criteria are considered definite cases of IE. Two features that are crucial with respect to blood cultures are persistent bacteremia and typical microorganisms. Viridans streptococcus, Streptococcus bovis and the members of HACEK group (Haemophilus species, Actinobacillus actinomycetemcomitans, Cardiobacte- 
TABLE 3

Definitions of terminology used in the Duke criteria

\begin{tabular}{l} 
Major criteria \\
Positive blood culture for infective endocarditis \\
Typical microorganism for infective endocarditis from two \\
separate blood cultures \\
- Viridans streptococci*, Streptococcus bovis, HACEK group, or \\
community-acquired Staphylococcus aureus or enterococci, \\
in the absence of a primary focus, or \\
Persistently positive blood culture, defined as recovery of a \\
microorganism consistent with infective endocarditis from: \\
blood cultures drawn more than $12 \mathrm{~h}$ apart, or \\
all of three or a majority of four or more separate blood \\
cultures, with first and last drawn at least $1 \mathrm{~h}$ apart \\
Evidence of endocardial involvement \\
Positive echocardiogram for infective endocarditis \\
oscillating intracardiac mass, on valve or supporting structures, \\
or in the path of regurgitant jets, or on implanted material, \\
in the absence of an alternative anatomic explanation; \\
abscess; or \\
new partial dehiscence of prosthetic valve \\
New valvular regurgitation (increase or change in pre-existing \\
murmur not sufficient) \\
Minor criteria \\
Predisopition: predisposing heart condition or intravenous drug \\
use \\
Fever: higher than $38.0^{\circ} \mathrm{C}$ (100.4 ${ }^{\circ}$ F) \\
Vascular phenomena: major arterial emboli, septic pulmonary \\
infarcts, mycotic aneurysm, intracranial hemorrhage, \\
conjunctival hemorrhages, Janeway lesions \\
Immunological phenomena: glomerulonephritis, Osler's nodes, \\
Roth's spots, rheumatoid factor \\
Microbiological evidence: positive blood culture but not meeting \\
major criterion as noted previously or serological evidence of \\
active infection with organism consistent with infective \\
endocarditis \\
not meeting major criterion as noted previously \\
\hline
\end{tabular}

${ }^{\dagger}$ Including nutritional variant strains; ${ }^{\ddagger}$ Excluding single positive cultures for coagulase-negative staphylococci and organisms that do not cause endocarditis. HACEK Haemophilus species, Actinobacillus actinomycetemcomitans, Cardiobacterium hominis, Eikenella species and Kingella kingae. Adapted from reference 2 with permission of Excerpta Medica Inc

rium hominis, Eikenella species and Kingella kingae) are common causes of IE and are infrequently isolated from blood cultures of patients without IE. On the other hand, Staphylococcus aureus and enterococcus may be typical endocarditis pathogens, but they also cause bacteremia in patients with confirmed extracardiac infections without IE, especially those that are acquired nosocomially. The Duke schema weighs persistent enterococcal and staphylococcal bacteremias as major criteria only when they are both community acquired and arise without an apparent primary focus of infection. Coagulase-negative staphylococcus can cause IE but is often isolated from blood culture usually because of contamination. This explains why persistent bacteremia is a key feature of these criteria.

The echocardiographic manifestations of IE include discrete, echogenic oscillating intracardiac masses located at sites of endocardial injury, periannular abscess and new dehiscence of a prosthetic valve. Based on the authors' clinical
TABLE 4

Comparison of clinical diagnosis of infective endocarditis using von Reyn criteria and Duke criteria in the pathologically proven cases from four independent centres $(2,4-6)$

\begin{tabular}{lcccc}
\hline & \multicolumn{4}{c}{ Duke criteria } \\
von Reyn criteria & Definite & Possible & Rejected & Total (\%) \\
\hline Probable & 88 & 5 & 0 & $93(57)$ \\
Possible & 32 & 5 & 0 & $36(23)$ \\
Rejected & 10 & 21 & 2 & $33(20)$ \\
Total (\%) & $130(80)$ & $31(19)$ & $2(1)$ & $163(100)$ \\
\hline
\end{tabular}

TABLE 5

Comparison of clinical diagnosis in patients evaluated for diagnosis of infective endocarditis from four independent centres $(2,4-6)$ using von Reyn criteria and Duke criteria excluding pathologically proven cases

\begin{tabular}{lcccc}
\hline & \multicolumn{4}{c}{ Duke criteria } \\
von Reyn criteria & Definite & Possible & Rejected & Total (\%) \\
\hline Probable & 116 & 13 & 12 & $141(30)$ \\
Possible & 88 & 62 & 15 & $165(34)$ \\
Rejected & 18 & 93 & 58 & $169(36)$ \\
Total (\%) & $222(47)$ & $168(35)$ & $85(18)$ & $475(100)$ \\
\hline
\end{tabular}

experience, echocardiography does not appear to be sensitive enough to pick up fenestrations of valvular leaflets; however, identification of fenestrations should be as a major criterion. Nonspecific valve thickening is not evidence of echocardiographic definite IE. False positive echocardiographic features in prosthetic valves could be related to stitches to the valvular ring or noninfective paravalvular leak. New valvular regurgitation by auscultation, however, is considered a main criterion under the Duke classification.

Predisposing factors include patients who have had previous mechanical or tissue prosthetic valves, intracardiac instrumentation or intravenous drug use. The presence of an ischemic leg or focal neurological manifestation consistent with stroke belongs to the vascular phenomenon. These conditions must be confirmed radiologically. Splinter hemorrhages, subconjunctival hemorrhages and clubbing are considered too nonspecific, and, therefore, they are not included in this category. Immunological phenomena include immune complexmediated events such as glomerulonephritis, Osler's nodes and Roth's spots. Echocardiographic features of the Duke minor criteria are those that are suggestive of IE but are not mentioned in the major category; valvular thickening and nodules are examples of this. Microbiological features include bacteremia not due to the typical organisms causing IE or serological evidence of recent infection by a pathogen known to cause valvular lesions (eg, brucella, legionella, chlamydia or Coxiella burnettii), where routine blood cultures may not be able to isolate the pathogen. Bartonella species have been recently recognized as an important cause of apparent 'blood culture- 
TABLE 6

The Toronto Hospital experience with diagnosis of infective endocarditis (IE) from 1989 to 1995

\begin{tabular}{|c|c|c|}
\hline & \multicolumn{2}{|c|}{ Number of patients } \\
\hline $\begin{array}{l}\text { Patients with discharge diagnosis } \\
\text { of IE }\end{array}$ & \multicolumn{2}{|c|}{206} \\
\hline \multicolumn{3}{|l|}{ Using Duke criteria: } \\
\hline Definite IE & \multicolumn{2}{|r|}{183} \\
\hline Probable IE & \multicolumn{2}{|r|}{23} \\
\hline Rejected IE & \multicolumn{2}{|r|}{0} \\
\hline \multicolumn{3}{|l|}{$\begin{array}{l}\text { Among } 183 \text { patients who fulfilled } \\
\text { definite Duke criteria: }\end{array}$} \\
\hline Age (years) & \multicolumn{2}{|c|}{$50.7 \pm 17.8$} \\
\hline Sex & \multicolumn{2}{|c|}{68 females } \\
\hline \multicolumn{3}{|l|}{ Clinical diagnosis of IE with: } \\
\hline Two major criteria & \multicolumn{2}{|c|}{152 patients } \\
\hline $\begin{array}{l}\text { One major and three minor } \\
\text { criteria }\end{array}$ & \multicolumn{2}{|c|}{30 patients } \\
\hline \multirow[t]{3}{*}{ Five minor criteria } & \multicolumn{2}{|c|}{2 patients } \\
\hline & \multicolumn{2}{|c|}{ Number of patients } \\
\hline & $\begin{array}{l}\text { Native valve } \\
(\%)\end{array}$ & $\begin{array}{c}\text { Prosthetic value } \\
(\%)\end{array}$ \\
\hline Number of patients & $126(100)$ & $57(100)$ \\
\hline \multicolumn{3}{|l|}{ Major criteria } \\
\hline Positive blood culture & $125(98)$ & $50(88)$ \\
\hline $\begin{array}{l}\text { Typical echocardiographic } \\
\text { features }\end{array}$ & $114(90)$ & $45(79)$ \\
\hline \multicolumn{3}{|l|}{ Minor criteria } \\
\hline Predisposition & $62(49)$ & $57(100)$ \\
\hline Temperature higher than $38^{\circ} \mathrm{C}$ & $110(87)$ & $52(91)$ \\
\hline Vascular phenomenon & $35(28)$ & $13(23)$ \\
\hline Immunological phenomenon & $18(14)$ & $6(11)$ \\
\hline Minor echocardiogram features & $6(5)$ & $9(16)$ \\
\hline Minor microbiological features & 0 & $1(2)$ \\
\hline \multicolumn{3}{|l|}{ Organisms } \\
\hline Staphylococcus aureus & $39(31)$ & $13(23)$ \\
\hline Staphylococcus epidermis & $8(6)$ & $11(19)$ \\
\hline Viridans streptococcus & $45(36)$ & $11(19)$ \\
\hline Other streptococcal species & $23(11)$ & $6(11)$ \\
\hline HACEK group & $3(2)$ & $4(7)$ \\
\hline Gram-positive bacilli & $3(2)$ & $1(2)$ \\
\hline Gram-negative bacilli & $3(2)$ & $4(7)$ \\
\hline Gram-negative anerobes & $1(1)$ & 0 \\
\hline Negative blood cultures & $1(1)$ & $7(12)$ \\
\hline
\end{tabular}

HACEK Haemophilus species, Actinobacillus actinomycetemcomitans, Cardiobacterium hominis, Eikenella species and Kingella kingae

negative' endocarditis in several reports (4-6), especially in patients who are alcoholics or are homeless. At this time, isolation of these organisms fulfils a minor Duke criterion.

\section{EVALUATION OF THE DUKE CRITERIA}

In addition to the internal evaluation by the Duke Endocarditis Service, the recently proposed criteria were also evaluated independently by several other centres, namely the University of Southern California in Los Angeles (7), the Uni-
TABLE 7

Clinical course of patients with infective endocarditis at The Toronto Hospital

\begin{tabular}{lcc}
\hline & Native valve & $\begin{array}{c}\text { Prosthetic } \\
\text { valve }\end{array}$ \\
\hline $\begin{array}{l}\text { Mean duration from the onset of } \\
\text { symptoms to the time of diagnosis } \\
\text { (range } 1 \text { to } 210 \text { days) }\end{array}$ & 33 days & 29 days \\
$\begin{array}{l}\text { Mean duration of hospital stay } \\
\text { (ranged } 1 \text { to } 281 \text { days) }\end{array}$ & 30 days & 40 days \\
$\begin{array}{l}\text { Number of patients who proceeded } \\
\text { with TEE after TTE }\end{array}$ & $74(59 \%)$ & $37(65 \%)$ \\
$\begin{array}{l}\text { Number of patients who had TEE } \\
\text { without TTE }\end{array}$ & $12(10 \%)$ & $11(19 \%)$ \\
$\begin{array}{l}\text { Number of patients suffered from at } \\
\text { least one embolic event before un- } \\
\text { dergoing echocardiography }\end{array}$ & $36(29 \%)$ & $6(11 \%)$ \\
$\begin{array}{l}\text { Outcome } \\
\text { Requiring urgent cardiac surgery }\end{array}$ & $63(50 \%)$ & $27(47 \%)$ \\
$\begin{array}{l}\text { Cerebral infarct } \\
\text { Systemic embolism }\end{array}$ & $26(21 \%)$ & $3(5 \%)$ \\
Pulmonary embolism & $24(19 \%)$ & 0 \\
$\begin{array}{l}\text { Congestive heart failure } \\
\text { Death }\end{array}$ & $4(3 \%)$ & $1(2 \%)$ \\
\hline TEE Transesophageal echocardiogram; TTE Transthoracic echocardiogram
\end{tabular}

versity of Nancy Medical Center in Vandoeuvre cedex, France (8) and the University of Alberta (9). Summarizing the results reported from the four centres, a total of 638 patients with suspected IE were evaluated; 163 had IE proven pathologically and 475 did not (Tables 4,5). Calculation of sensitivity and specificity was difficult because the pathology (the gold standard) is not available on all patients. However, the conclusion drawn from these studies is that, using the new set of criteria, more cases would be classified as definite versus probable, possible or even rejected compared with the old criteria. Dodds et al (10) showed that the negative predictive value of the new criteria was as high as $98 \%$ during a threemonth follow-up of patients in whom the diagnosis of IE was rejected. In this study, no new cases were found in these patients except one case where the patient's autopsy revealed evidence that might represent missed IE. Thus, the Duke criteria are more sensitive than the old criteria because they include more patients in the positive test category. Moreover, a patient failing to satisfy the criteria for probable IE is very unlikely to have IE.

\section{THE TORONTO HOSPITAL EXPERIENCE}

Patient selection: The Toronto Hospital is a tertiary referral centre. The authors' experience with IE from 1989 to 1995 matched the data reported from other centres. Two hunderd and six cases were identified based on a discharge diagnosis of IE reported to the hospital registry. The hospital charts were reviewed retrospectively, and each case was classified as 'def- 
inite', 'probable' or 'rejected' based on the Duke criteria (Table 6).

Results: The clinical characteristics of the patients, including the causative organisms and the course in the hospital, are presented in Tables 6 and 7. One hundred and eighty-three and 23 cases were labelled as 'definite IE' and 'probable IE', respectively, under the new criteria (ie, no cases were rejected under the new criteria). There were 90 'definite IE' patients and two in the 'probable IE' group who underwent cardiac surgery. The two patients who belonged to the 'probable' group presented with one major (positive blood culture) and two minor criteria; however, during surgery only one patient was identified as having active infective endocarditis.

of note, $98 \%$ of the patients with native valve endocarditis had typical blood cultures, and a large portion of patients also had typical echocardiographic features of IE (Table 6). This implies that in the absence of bacteremia and no preceding antibiotic use, patients who have native valves and present with other features of IE are in fact unlikely to have endocarditis.

In addition, more than half of the patients proceeded to transesophageal echocardiography after transthoracic scanning. Moreover, a higher percentage of patients with prosthetic valves had transesophageal echocardiogram as the initial radiological test compared with native valve group, probably because of a high pretest suspicion (presence of positive blood culture and prosthesis) and poor acoustics of prosthesis using transthoracic method.

\section{REFERENCES}

1. von Reyn CF, Levy BS, Arbeith RD, Friedland G, Crumpacker CS. Infective endocarditis: an analysis based on strict case definitions. Ann Intern Med 1981;94:505-17.

2. Durack DT, Lukes AS, Bright DK. New criteria for diagnosis of infective endocarditis: utilization of specific echocardiographic findings. Am J Med 1994;96:200-9.

3. Guidelines for the diagnosis of rheumatic fever. Jones' criteria, 1992 update. Special Writing Group of the Committee on Rheumatic Fever, Endocarditis, and Kawasaki Disease of the Council on Cardiovascular Disease in the Young of the American Heart Association. JAMA 1992;268:2069-73.

4. Breathnach AS, Hoare JM, Eykyn SJ. Culture-negative endocarditis: contribution of bartonella infections. Heart 1997;77:474-6.

5. Raoult D, Fournier PE, Drancourt M, et al. Diagnosis of 22
Among patients with prosthetic valves, the majority had typical microorganisms cultured from their blood, but typical echocardiographic features were seen less frequently than in those with native valve IE. This is likely due to the difficulty of detecting vegetations in the presence of valvular prosthesis, mainly as a result of acoustic shadowing on echocardiograms. Our anecdotal experience suggested that patients whose blood cultures were positive for typical organisms and had a history of previous prosthetic valve surgery should be treated with full antibiotic therapy, as if endocarditis was present.

\section{CONCLUSIONS}

Infective endocarditis is difficult to diagnose with certainty. Clinicians often face a diagnostic dilemma when a patient presents with some symptoms suggesting IE, especially in cases when patients with prosthetic heart valves present with febrile illness. However, careful examination using the guidelines from the Duke criteria may assist clinicians in making a diagnosis of IE with greater certainty, and thus the decision regarding antibiotics administration may be made more appropriately. In our centre, the Duke criteria identified most patients with definite endocarditis except for one patient who subsequently was proven to have IE during surgery. The Duke criteria, therefore, provide a more satisfactory set of guidelines than the previous guidelines for diagnosing IE and determining which patients should be treated.

new cases of bartonella endocarditis. Ann Intern Med 1996;125:646-52.

6. Spach DH, Kanter AS, Daniels NA, et al. Bartonella (Rochalimaea) species as a cause of apparent 'culture-negative' endocarditis. Clin Infect Dis 1995;20:1044-7.

7. Bayer AS, Ward JI, Ginzton LE, Shapiro SM. Evaluation of new clinical criteria for the diagnosis of infective endocarditis. Am J Med 1994;96:211-9.

8. Hoen B, Selton-Suty C, Danchin N, et al. Evaluation of the Duke criteria versus the Beth Israel criteria for the diagnosis of infective endocarditis. Clin Infect Dis 1995;21:905-9.

9. Sandre RM, Shafran SD. Infective endocarditis: Review of 135 cases over 9 years. Clin Infect Dis 1996;22:276-86.

10. Dodds GA, Sexton DJ, Durack DT, et al. Negative predictive value of the Duke criteria for infective endocarditis. Am J Cardiol 1996;77:403-7. 


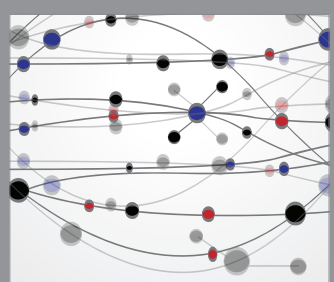

The Scientific World Journal
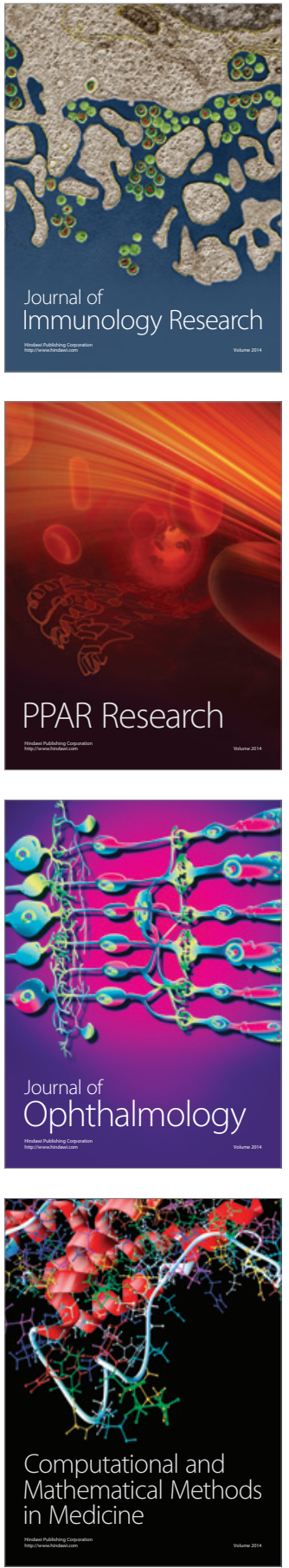

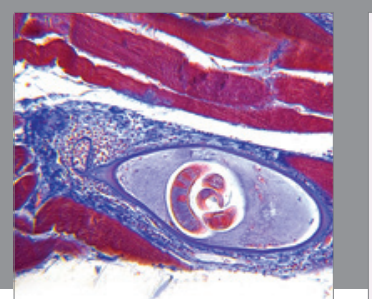

Gastroenterology Research and Practice

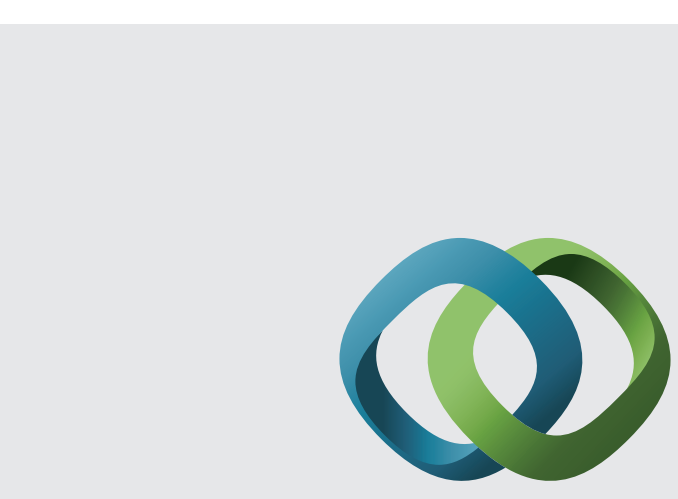

\section{Hindawi}

Submit your manuscripts at

http://www.hindawi.com
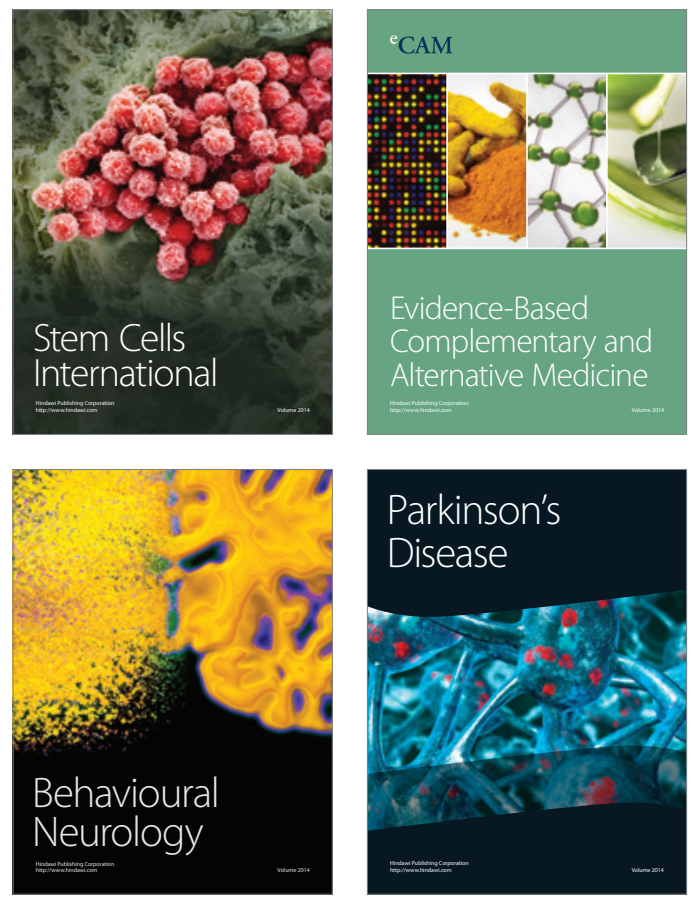
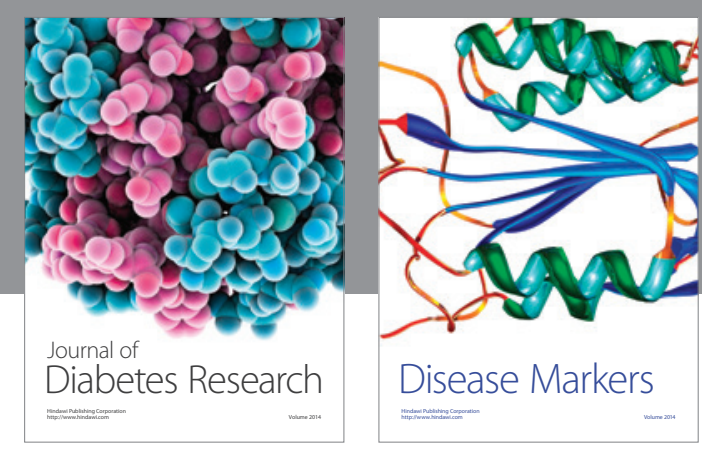

Disease Markers
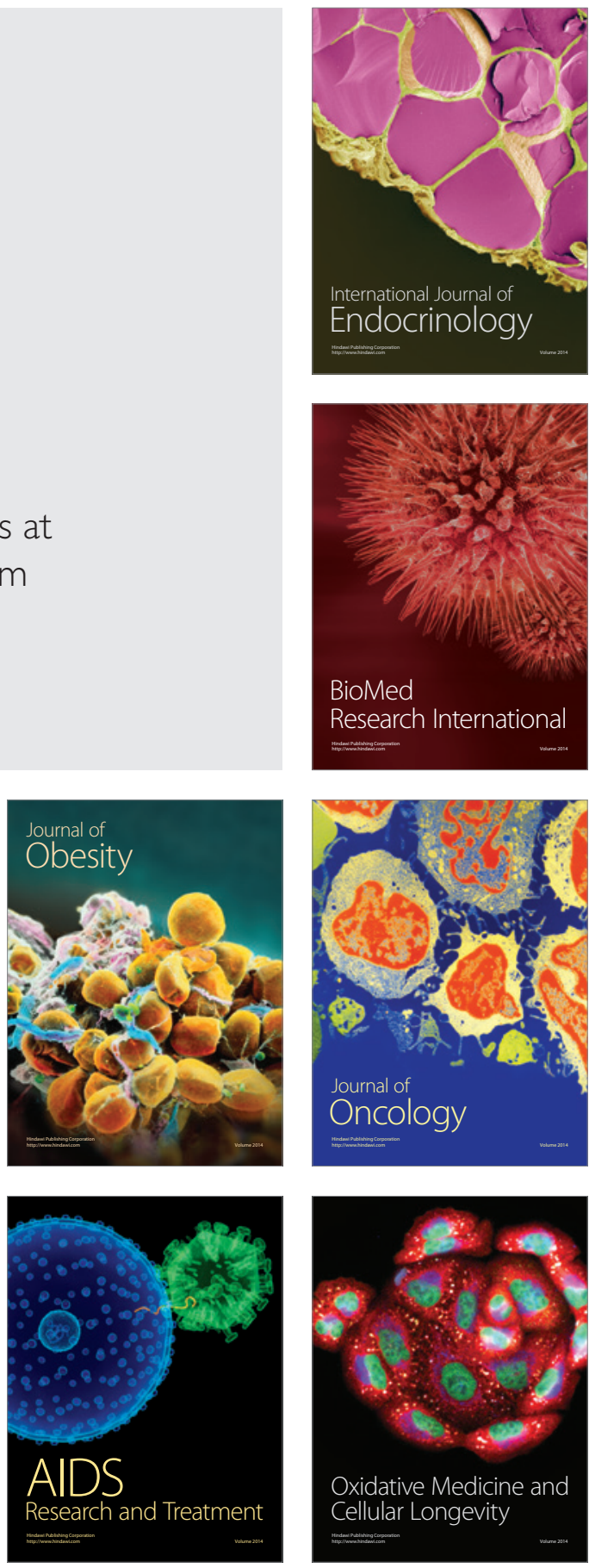